\title{
Compassionate use of delamanid in combination with bedaquiline for the treatment of multidrug-resistant tuberculosis
}

To the Editor:

Patients with multidrug-resistant (MDR) tuberculosis (TB), in particular those with pre-extensively drug-resistant (pre-XDR) and extensively drug-resistant (XDR)-TB, and those that fail standard second-line therapy, are difficult to treat and have poor long-term outcomes [1]. To address this unmet medical need, there is strong interest in exploring the combined use of delamanid and bedaquiline, the only two anti-TB drugs approved for the treatment of pulmonary MDR-TB in the last 40 years, as their novel mechanisms of action may offer treatment alternatives for patients who have developed resistance or non-tolerability to existing anti-TB drugs $[2,3]$. Despite the initial regulatory approvals of bedaquiline and delamanid in 2012 and 2014, respectively, global usage of both drugs in combination with one another remains limited in part due to the uncertainty around the safety and efficacy of such a combination regimen. Hence, there is an urgent need for programmatic data to better understand the "real-world" use of these two medicines used together in MDR-TB patients.

As part of a global access initiative, Otsuka Pharmaceutical Co., Ltd., in coordination with the European Respiratory Society (ERS)/World Health Organization (WHO) TB Consilium and Médecins Sans Frontières/Partners in Health (MSF-PIH), established its first Compassionate Use (CU) programme in 2014 to provide access to delamanid, at no cost, for patients with limited treatment options [4]. In 2016, the programme was modified to allow for the combined use of delamanid plus bedaquiline under specific conditions. We present here the early safety and efficacy outcomes of patients enrolled in this programme receiving delamanid and bedaquiline concomitantly along with other anti-TB drugs for the treatment of MDR-TB.

Requests for CU were submitted to the ERS/WHO TB Consilium or MSF-PIH for review, followed by the final review and approval of the Otsuka CU committee as previously described [4]. Patients receiving combined delamanid plus bedaquiline were required to meet the following inclusion criteria: signed informed consent by patient (or by parent or legal guardian for minors); MDR-TB with limited therapeutic options; unable to participate in a delamanid clinical trial; age $\geqslant 6$ years and weight $>20 \mathrm{~kg}$; able to receive treatment with a background regimen consistent with WHO guidelines at a centre experienced in MDR-TB management; and agreement to hospitalisation for the initial 4 months of combination therapy. Exclusion criteria were: prior exposure to delamanid; albumin $<2.8 \mathrm{~g} \cdot \mathrm{dL}^{-1}$; electrolyte imbalances; baseline QT interval (QTc) corrected for heart rate by Fridericia's method $>450 \mathrm{~ms}$; fewer than two active or likely to be active drugs (not counting delamanid) to be included in the proposed background regimen based on recent drug susceptibility testing (DST) results and/or treatment history; concurrent use of strong cytochrome P450 3A4 inhibitors or other anti-TB agents in development; and hypersensitivity to delamanid.

Patients accepted for CU were treated with delamanid $100 \mathrm{mg}$ twice daily (or $50 \mathrm{mg}$ twice daily for patients with weight $<35 \mathrm{~kg}$ ) for 24 weeks in combination with a background regimen designed in

@ERSpublications

Updated data from the Otsuka compassionate use programme show that regimens combining delamanid and bedaquiline appear effective in MDR-TB cases with limited treatment options http:// ow.ly/zdE330mlkLN

Cite this article as: Hafkin J, Hittel N, Martin A, et al. Compassionate use of delamanid in combination with bedaquiline for the treatment of multidrug-resistant tuberculosis. Eur Respir J 2019; 53: 1801154 [https://doi.org/10.1183/13993003.01154-2018]. 
accordance with WHO guidelines and local guidelines. Other drugs, including bedaquiline, were administered as per the treating physician's discretion with respect to dose and duration. Patient management, including length of and changes to the regimen, as well as frequency and duration of follow-up, were made by the treating physicians (weekly ECGs were recommended for all patients receiving delamanid combined with bedaquiline for the duration of co-administration). Pharmacovigilance (PV) training of treating physicians was performed prior to treatment initiation and safety data were collected throughout the delamanid treatment period. Culture-negative status was defined as having one or more negative cultures within 24 weeks after starting delamanid without subsequent positive cultures within this period.

Baseline data from all cases (e.g. demographic information, baseline culture status, DST results, disease type, presence of cavities, drug resistance category, history of prior treatment, and comorbidities) were prospectively collected via a standardised patient access forms (completed by the treating physician) and recorded into an Excel spreadsheet (Microsoft, Redmond, WA, USA) by the Otsuka CU team for further analyses. All adverse events were prospectively captured, and reported to the Otsuka PV team during the course of therapy and assessed for seriousness, severity and association (i.e. causality) with delamanid. In addition, culture information during the 24-week period, confirmation of the regimen given at baseline, use of delamanid including temporary interruptions, permanent withdrawals from therapy and completion of a treatment course were collected by Otsuka after the initiation of delamanid therapy. Descriptive statistics were performed for all patients who initiated treatment with delamanid (in combination with bedaquiline) prior to February 2018. Categorical data were reported as counts and proportions, and continuous data were reported as means and/or medians and ranges.

From February 2014 until February 2018, 238 CU requests for delamanid were received, 199 approved, five were pending review, 11 did not meet inclusion criteria and 23 were withdrawn by the requestor prior to review. Of the 199 approved, 28 requests were withdrawn by the requestor prior to delamanid start, two were pending delamanid shipment and 169 patients initiated delamanid treatment including 84 that initiated delamanid treatment with a bedaquiline-containing background regimen for $\geqslant 1$ day. Of these 84 patients, 58 completed 24 weeks of treatment with delamanid, 12 were still on treatment, one permanently discontinued treatment (patient was non-cooperative with the clinic staff), 10 had died prior to completing delamanid (median of 39 days (range 3-113 days) after delamanid initiation) and three had unknown completion status. Of the 84 patients treated with delamanid and bedaquiline concurrently, $19.0 \%$ (16 out of 84 ) started bedaquiline prior to starting delamanid (median of 110 days (range 25331 days) prior to the start of delamanid), $79.8 \%$ (67 out of 84 ) were naïve to both drugs at the time of their initiation, and $1.2 \%$ (one out of 84 ) had unknown treatment history prior to receiving both drugs.

Demographic and clinical characteristics of the 84 patients who initiated treatment with delamanid in the context of a background regimen containing bedaquiline are provided in table 1 . The mean age and weight were 37 years and $55 \mathrm{~kg}$, respectively. $7.1 \%$ (six out of 84 ) were $<18$ years and $67.0 \%$ (56 out of 84 ) were male. $90.5 \%$ (76 out of 84 ) of patients originated from sub-Saharan Africa, 3.6\% (three out of 84 ) from Asia and $6.0 \%$ (five out of 84 ) from the Americas. $96.4 \%$ (81 out of 84 ) had pulmonary disease alone, $2.4 \%$ (two out of 84 ) had extrapulmonary disease plus pulmonary disease (one with central nervous system, lymph node and pulmonary disease; and one with spinal plus pulmonary disease) and 1.2\% (one out of 84) extrapulmonary disease alone (isolated lymph node disease). 88.1\% (74 out of 84) of patients were culture positive at referral. Of the six children enrolled in the $\mathrm{CU}$ project, both received delamanid and bedaquiline, and five had pulmonary $\mathrm{TB}$ and one had both pulmonary plus extrapulmonary involvement (lymphadenitis and cerebral disease). Their ages at the time of enrolment were 8, 11, 12, 13, 14 and 14 years.

88.1\% (74 out of 84 ) patients overall had received a previous course (or courses) of TB treatment (all with both first- and second-line drugs), while only $11.3 \%$ (10 out of 84 ) had delamanid added to their initial, current TB treatment course. In terms of baseline resistance status, $73.8 \%$ (62 out of 84) had XDR-TB, $21.4 \%$ (18 out of 84 ) had pre-XDR-TB (i.e. MDR-TB with additional resistance to either a fluoroquinolone or a second-line injectable agent) and 4.8\% (4 out of 84 ) had MDR-TB.

$54.8 \%$ (46 out of 84 ) of patients had concomitant HIV co-infection and among the 34 out of $46(73.9 \%)$ with available $\mathrm{CD}^{+} \mathrm{T}$-cell counts at baseline, the mean value was 320 cells per $\mathrm{mm}^{3}$ (range $62-839$ cells per $\mathrm{mm}^{3}$ ). In addition, 44 out of $46(95.7 \%)$ were on antiretroviral (ARV) therapy, one out of $46(2.2 \%)$ was not on ARV therapy and ARV treatment status was unknown for one out of $46(2.2 \%)$.

In terms of other comorbidities reported to have occurred in at least two or more individuals at baseline, six out of $84(7.1 \%)$ had diabetes, four out of 84 (4.8\%) had hearing impairment, three out of $84(3.6 \%)$ had renal dysfunction, three out of $84(3.6 \%)$ had visual impairment, three out of $84(3.6 \%)$ had substance abuse and three out of 84 (3.6\%) had hypertension. 
TABLE 1 Demographic and clinical characteristics of compassionate use patients initiating delamanid plus bedaquiline prior to February 2018

\begin{tabular}{|c|c|}
\hline Age years & $37(8-63)$ \\
\hline Weight kg & $55(22-110)$ \\
\hline Body mass index $\mathrm{kg} \cdot \mathrm{m}^{-2}$ & $19.7(12.0-31.5)$ \\
\hline \multicolumn{2}{|l|}{ Sex } \\
\hline Female & $28(33.0 \%)$ \\
\hline Male & $56(67.0 \%)$ \\
\hline \multicolumn{2}{|l|}{ Region } \\
\hline Europe & $0(0 \%)$ \\
\hline Asia & $3^{\S}(3.6 \%)$ \\
\hline Sub-Saharan Africa & $76^{f}(90.5 \%)$ \\
\hline Americas & $5^{\# \#}(6.0 \%)$ \\
\hline \multicolumn{2}{|l|}{ Disease type } \\
\hline Pulmonary disease alone & $81(96.4 \%)$ \\
\hline Pulmonary and extrapulmonary disease & $2(2.4 \%)$ \\
\hline Extrapulmonary disease alone & $1(1.2 \%)$ \\
\hline \multicolumn{2}{|l|}{ Cavitary disease } \\
\hline No & $6(7.1 \%)$ \\
\hline Yes & $53(63.1 \%)$ \\
\hline Unknown & $25(29.8 \%)$ \\
\hline \multicolumn{2}{|l|}{ Resistance type } \\
\hline MDR & $4(4.8 \%)$ \\
\hline Pre-XDR & $18(21.4 \%)$ \\
\hline XDR & $62(73.8 \%)$ \\
\hline \multicolumn{2}{|l|}{ History of prior TB treatment } \\
\hline Yes & $74(88.1 \%)$ \\
\hline No & $10(11.9 \%)$ \\
\hline \multicolumn{2}{|l|}{ HIV status } \\
\hline Positive & $46(54.8 \%)$ \\
\hline Negative & $36(42.9 \%)$ \\
\hline Unknown & $2(2.4 \%)$ \\
\hline \multicolumn{2}{|l|}{ Culture status at referral } \\
\hline Positive & $75(89.3 \%)$ \\
\hline Negative & $9(10.7 \%)$ \\
\hline \multicolumn{2}{|c|}{$\begin{array}{l}\text { Drugs used in optimised background regimens among } \\
\text { patients receiving delamanid and bedaquiline }\end{array}$} \\
\hline Linezolid & $77(91.7 \%)$ \\
\hline Pyrazinamide & $68(81.0 \%)$ \\
\hline Clofazimine & $66(78.6 \%)$ \\
\hline Para-aminosalicylic acid & $63(75.0 \%)$ \\
\hline Fluoroquinolones & $53(63.1 \%)$ \\
\hline Ethionamide/prothionamide & $30(35.7 \%)$ \\
\hline Carbapenems & $30(35.7 \%)$ \\
\hline Cycloserine/terizadone & $28(33.3 \%)$ \\
\hline Ethambutol & $15(17.9 \%)$ \\
\hline Second-line injectable & $14(16.7 \%)$ \\
\hline High-dose isoniazid & $11(13.1 \%)$ \\
\hline \multicolumn{2}{|c|}{ Culture status at 24 weeks among delamanid treatment completers ${ }^{\eta,+}$} \\
\hline Culture negative & $51(87.9 \%)$ \\
\hline Culture positive & $4(6.9 \%)$ \\
\hline Unknown or pending & $3(5.2 \%)$ \\
\hline
\end{tabular}

Data are presented as median (range), unless otherwise stated. $\mathrm{n}=84$ unless otherwise stated. MDR: multidrug-resistant; pre-XDR: pre-extensively drug resistant; XDR: extensively drug resistant; TB: tuberculosis. ${ }^{\#}: n=79 ;$ ๆ $54(93.1 \%)$ out of 58 were culture-positive at baseline; ${ }^{+}: n=58$; ${ }^{\S}$ : all in India; ${ }^{f}$ : one in Swaziland, one in Namibia and 74 in South Africa; ${ }^{\# \#}$ : one in the USA, one in Chile, one in Mexico and two in Peru.

The majority of patients received the following concomitant anti-TB agents (in addition to bedaquiline) during the 6-month delamanid treatment period: linezolid, $91.7 \%$ (77 out of 84 ); pyrazinamide, $81.0 \%(68$ out of 84 ); clofazimine, $78.6 \%$ (66 out of 84 ); para-aminosalicylic acid, $75.0 \%$ (63 out of 84 ); a fluoroquinolone, $63.1 \%$ (53 out of 84 ).

Of the 58 patients who completed 24 weeks of treatment with delamanid and bedaquiline plus a background regimen, $87.9 \%$ (51 out of 58) achieved culture-negative status at 24 weeks, $6.9 \%$ (four out of 58) 
remained culture positive at 24 weeks and 5.2\% (three out of 58) had results pending or unknown. Limiting the analysis to only the 40 patients with XDR-TB yielded similar results: $85.0 \%$ (34 out of 40) achieved culture-negative status at 24 weeks, $7.5 \%$ (three out of 40 ) remained culture positive at 24 weeks and $7.5 \%$ (three out of 40 ) had unknown culture status.

These results compare favourably to those seen in the population of patients who did not receive bedaquiline along with delamanid. Among these patients, 52 out of $65(80.0 \%)$ had negative culture status at 24 weeks, 11 out of $65(16.9 \%)$ had positive culture status and two out of $65(3.1 \%)$ had unknown culture status.

In terms of those patients who had prior exposure to either clofazimine or bedaquiline prior to starting combination delamanid plus bedaquiline, the 6-month culture responses were as follows: among the five patients who had prior bedaquiline exposure and completed 24 weeks of delamanid, three out of five (60.0\%) achieved negative cultures, one out of five $(20.0 \%)$ did not achieve negative cultures and one out of five $(20.0 \%)$ had unknown culture status; among the 49 patients who completed 24 weeks of delamanid and had prior clofazimine exposure, 44 out of 49 (89.8\%) achieved negative cultures, three out of 49 (6.1\%) did not achieve negative cultures and two out of 49 (4.1\%) had unknown culture status. In terms of the patients who had no prior exposure to either clofazimine or bedaquiline, the outcomes were as follows: among the 52 patients who had no prior bedaquiline exposure and completed 24 weeks of delamanid, 47 out of $52(90.4 \%)$ achieved negative cultures, three out of $52(5.8 \%)$ did not achieve negative cultures and two out of $52(3.8 \%)$ had unknown culture status; among the eight patients who had no prior clofazimine exposure, six out of eight (75\%) achieved negative cultures, one out of eight (12.5\%) remained culture positive and one out of eight (12.5\%) had unknown culture status.

In terms of HIV status among the 6-month treatment completers, 35 out of 58 (60.3\%) were HIV positive, 21 out of $58(36.2 \%)$ were HIV negative and two out of 58 (3.4\%) had unknown HIV status. Among the 35 HIV-positive patients, 33 out of 35 (94.3\%) achieved culture negativity, one out of $35(2.9 \%)$ remained culture positive and one out of $35(2.9 \%)$ had an unknown culture status. Among the $21 \mathrm{HIV}$-negative patients, 16 out of $21(76.2 \%)$ achieved culture negativity, three out of 21 (14.3\%) remained culture positive and two out of 21 (9.5\%) had an unknown culture status.

Finally, among the six children enrolled in the CU project who received both delamanid and bedaquiline, the five with pulmonary disease were culture negative at the end of delamanid treatment and the one child with additional extrapulmonary involvement was clinically doing well at the end of treatment.

QT prolongation of any duration was reported in five (6.0\%) patients; however, only one (1.2\%) patient was reported to have a corrected QTc of $>500 \mathrm{~ms}$. In this case, the patient was receiving clofazimine in addition to delamanid and bedaquiline (i.e. all at the same time). Initial QT prolongation in this patient (i.e. QTc $486 \mathrm{~ms}$ ) led to temporary withdrawal of bedaquiline (days 12-16 of delamanid treatment); however, bedaquiline was successfully re-introduced (in combination with verapamil) in consultation with the ERS consilium. Subsequently, the treating physician chose to permanently discontinue clofazimine at week 6 due to QTc $>500 \mathrm{~ms}$, which eventually resolved (i.e. to levels $<500 \mathrm{~ms}$ ) without further regimen change [5-7]. Despite the QT elevation, the patient completed his course of delamanid without any associated clinical sequelae.

Amongst the 10 patients receiving both delamanid and bedaquiline who died prior to completing 24 weeks of treatment with delamanid, the causes of death were as follows: progressive TB (one patient), pulmonary embolism (one patient), myocardial infarction (one patient), sepsis secondary to gangrene (one patient), adrenal crisis and TB progression (one patient), haemoptysis (two patients), and unknown (three patients). None of these deaths were described by the treating clinician as likely to have been caused by delamanid, but rather due to other causes (i.e. predominantly underlying disease processes and/or progression). Of note, among the 10 patients who died, five were co-infected with HIV.

This analysis represents the largest published cohort of MDR-TB patients $(n=84)$ treated with delamanid plus bedaquiline. Although the data remain preliminary, the majority $(87.9 \%)$ of patients in the CU programme who completed 24 weeks of delamanid treatment had encouraging outcomes (i.e. negative 24-week sputum culture status), which is consistent with results from other published reports on combined use of delamanid and bedaquiline [5-11].

Moreover, these results are comparable to a recent systematic review by PonTALI et al. [11], which included 87 patients receiving delamanid and bedaquiline either concurrently or sequentially and reported culture conversion rates of $81.4 \%$. Similar to those patients included in the systematic review, the majority of patients in the present $\mathrm{CU}$ cohort had XDR-TB and were retreatment cases. Important differences, however, include the fact that the majority of patients in this CU cohort were co-infected with HIV, and that this analysis includes cases of combination delamanid plus bedaquiline use in children. 
As noted previously, early sputum culture conversion is a positive predictor of favourable long-term treatment outcomes $[12,13]$. The results are notable given that the majority of patients in this cohort had XDR-TB, extensive prior TB treatment history, cavitary disease and HIV co-infection; all features known to be associated with poor treatment outcomes [14]. Despite the disease severity and presence of these comorbidities, safety data amongst the patients initiating treatment with delamanid and bedaquiline concomitantly did not reveal any concerns not previously identified in the delamanid or bedaquiline clinical development programmes.

An evaluation of the safety and/or efficacy of the combination of both medicines is underway in two ongoing, prospective, randomised clinical trials: AIDS Clinical Trials Group 5343 (www.clinicaltrials.gov identifier number NCT02583048) and EndTB (NCT02754765). However, data from these studies will likely not be available until 2019 and 2021, respectively.

The absence of a control arm, incomplete information on duration of and changes to the background regimen (following delamanid initiation), and the lack of long-term treatment outcomes (including relapse data) are important limitations of this analysis. Nevertheless, these results are important as they represent a large, diverse pool of patients who generally would not have qualified for enrolment in a clinical trial due to the severe nature of their disease. In summary, these results are encouraging, and provided that a treatment site is experienced in MDR-TB management and measures are in place for adequate treatment monitoring [15], the evidence appears to yield a favourable benefit-risk profile to support the combined use of delamanid and bedaquiline in MDR-TB (including XDR-TB) patients with limited treatment options.

Jeffrey Hafkin ${ }^{1}$, Norbert Hittel ${ }^{2}$, Alexandra Martin ${ }^{2}$ and Rajesh Gupta ${ }^{1}$

${ }^{1}$ Otsuka Pharmaceutical Development and Commercialization, Inc., Rockville, MD, USA. ${ }^{2}$ Otsuka Novel Products GmbH, Munich, Germany.

Correspondence: Jeffrey Hafkin, Otsuka Novel Products, 2440 Research Blvd, Rockville, MD 20850, USA. E-mail: jeffrey. hafkin@otsuka-us.com

Received: June 202018 | Accepted after revision: Oct 122018

Acknowledgements: The authors would like to thank Natasa Lazarevic, Marion Lozano and Lusine Breitscheidel (Otsuka Pharmaceutical Co. Ltd) for their generous assistance in the preparation and review of data utilised for this analysis.

Conflict of interest: J. Hafkin is an employee of Otsuka Pharmaceutical Co. Ltd, which discovered, developed, manufactures and distributes delamanid. N. Hittel is an employee of Otsuka Pharmaceutical Co. Ltd, which discovered, developed, manufactures and distributes delamanid. A. Martin is an employee of Otsuka Pharmaceutical Co. Ltd, which discovered, developed, manufactures and distributes delamanid. R. Gupta is an employee of Otsuka Pharmaceutical Co. Ltd, which discovered, developed, manufactures and distributes delamanid.

\section{References}

1 Dheda K, Limberis JD, Pietersen E, et al. Outcomes, infectiousness, and transmission dynamics of patients with extensively drug-resistant tuberculosis and home-discharged patients with programmatically incurable tuberculosis: a prospective cohort study. Lancet Respir Med 2017; 5: 269-281.

2 Dedicoat M. Using bedaquiline and delamanid in combination and safely. Int J Tuberc Lung Dis 2016; $20: 1282$.

3 World Health Organization. WHO best-practice statement on the off-label use of bedaquiline and delamanid for the treatment of multidrug-resistant tuberculosis, 2017. http://apps.who.int/iris/handle/10665/258941 Date last accessed: April 17, 2018.

4 Hafkin J, Hittel N, Martin A, et al. Early outcomes in MDR-TB and XDR-TB patients treated with delamanid under compassionate use. Eur Respir J 2017; 50: 1700311.

5 Tadolini M, Lingtsang RD, Tiberi S, et al. First case of extensively drug-resistant tuberculosis treated with both delamanid and bedaquiline. Eur Respir J 2016; 48: 935-938.

6 Tadolini M, Lingtsang RD, Tiberi S, et al. First case, concerns and challenges of treatment of severe XDR-TB with both delamanid and bedaquiline. Eur Respir J 2016; 48: 1527-1529.

7 Maryandyshev A, Pontali E, Tiberi S, et al. Bedaquiline and delamanid combination treatment of 5 patients with pulmonary extensively drug-resistant tuberculosis. Emerg Infect Dis 2017; 23: 1718-1721.

8 Ferlazzo G, Mohr E, Laxmeshwar C, et al. Early safety and efficacy of the combination of bedaquiline and delamanid for the treatment of patients with drug-resistant tuberculosis in Armenia, India, and South Africa: a retrospective cohort study. Lancet Infect Dis 2018; 18: 536-544.

9 Guglielmetti L, Barkane L, Le Du D, et al. Safety and efficacy of exposure to bedaquiline-delamanid in multidrug-resistant tuberculosis: a case series from France and Latvia. Eur Respir J 2018; 51: 1702550.

10 Tae Kim C, Kim T-O, Shin H-J, et al. Bedaquiline and delamanid for the treatment of multidrug-resistant tuberculosis: a multicentre cohort study in Korea. Eur Respir J 2018; 51: 1702467.

11 Pontali E, Sotgiu G, Tiberi S, et al. Combined treatment of drug-resistant tuberculosis with bedaquiline and delamanid: a systematic review. Eur Respir J 2018; 52: 1800934.

12 Kurbatova EV, Cegielski PJ, Lienhardt C, et al. Sputum culture conversion as a prognostic marker for end-of-treatment outcome in patients with multidrug-resistant tuberculosis: a secondary analysis of data from two observational cohort studies. Lancet Respir Med 2015; 3: 201-209.

13 Wells CD, Gupta R, Hittel N, et al. Long-term mortality assessment of multidrug-resistant tuberculosis patients treated with delamanid. Eur Respir J 2015; 45: 1498-1501. 
14 Migliori GB, Sotgiu G, Gandhi NR, et al. Drug resistance beyond extensively drug-resistant: individual patient data meta-analysis. Eur Respir J 2013; 42: 169-179.

15 Matteelli A, D’Ambrosio L, Centis R, et al. Compassionate and optimum use of new tuberculosis drugs. Lancet Infect Dis 2015; 16: 1131-1132.

Copyright @ERS 2019 